\title{
REPOSO Y GERMINACIÓN DE SEMILLAS DE ENTEROLOBIUM CYCLOCARPUM (FABACEAE): RESULTADOS DE UN ESTUDIO INÉDITO Y UN EXPERIMENTO FALLIDO
}

\author{
CARLOS O. MORALES \\ Escuela de Biología, Universidad de Costa Rica, 2060 San José, Costa Rica \\ Jardín Botánico Lankester, Universidad de Costa Rica, apdo. 1031-7050 Cartago, Costa Rica
}

\begin{abstract}
Seed dormancy and germination of Enterolobium cyclocarpum (Fabaceae): Results of an unpublished work and an unsuccessful experiment. In Costa Rica, it was determined that in order to reproduce the tree species E. cyclocarpum, selected seeds might weigh 0,7 to $1,0 \mathrm{~g}$. In general, the weightiest fruits have the greatest mass of viable seeds, so that these fruits might contain the better seeds to be selected. Some seeds of this species germinated after 13 years of dormancy. Now there is evidence that the seeds of a particular crop are predeterminate to germinate in different times, this fact revealing a mid- and long-term advantageous reproductive strategy.
\end{abstract}

ReSUMEN. En Costa Rica, se determinó que para reproducir la especie arbórea Enterolobium cyclocarpum (Fabaceae), las semillas seleccionadas deben tener una masa de 0,7 a 1,0 g. En general, mayor masa del fruto implica mayor masa de semillas viables, de modo que frutos más grandes podrán contener las mejores semillas. Algunas semillas de esta especie germinaron después de 13 años en reposo. Ahora existe evidencia de que las semillas de una cosecha dada están programadas para germinar en tiempos diferentes, lo que revela una estrategia reproductiva ventajosa a mediano y largo plazo.

PALABRAS ClAVe / Key words: Enterolobium cyclocarpum, Fabaceae, guanacaste, Ear tree, reposo de semillas, germinación, seed dormancy and germination, Costa Rica.

Generalidades. El guanacaste, Enterolobium cyclocarpum (Jacq.) Griseb. (Fabaceae), árbol nacional de Costa Rica, alcanza hasta $30 \mathrm{~m}$ de altura y desarrolla una copa muy extendida (en algunos casos, más de $20 \mathrm{~m}$ de ancho). Su ámbito natural de distribución va desde México, América Central y las Antillas hasta el norte de Sudamérica, pudiendo adaptarse a diversas zonas de vida, como el bosque tropical húmedo, el bosque tropical seco y el bosque muy húmedo premontano (Bertsch 1985, Camacho 1981, Holdridge et al. 1997, Quesada et al. 1997, Vásquez-Yanes \& Pérez-García 1977).

El fruto de este árbol es una vaina ancha, aplanada, curva e indehiscente. El nombre indígena guanacaste significa árbol de orejas y se refiere a la forma particular del fruto. Las semillas maduras bien desarrolladas tienen una testa muy dura e impermeable, de modo que pocas veces son atacadas por insectos. La dureza, la resistencia y la impermeabilidad notables de la testa en muchas leguminosas se deben a capas de esclereidas lignificadas. Las reservas contenidas en los cotiledones son principalmente proteínas y carbohidratos ( $c f$. Bertsch 1985, Duarte 1978, Record \& Hess 1949, Vásquez-Yanes \& Pérez-García 1977). Después de madurar, las semillas del guanacaste germinan tras las primeras lluvias de la siguiente estación lluviosa o permanecen en reposo. ¿Cuánto tiempo máximo tarda el reposo? Hasta ahora, no parece haber datos concretos.

Resumen de un estudio inédito. Realicé un estudio sobre fenología y reproducción ${ }^{1}$ (cuyos resultados no se publicaron) en Turrúcares, Alajuela, Costa Rica $\left[9^{\circ}\right.$ $58^{\prime} \mathrm{N}, 84^{\circ} 19^{\prime} \mathrm{O}$, bosque tropical húmedo con transición a premontano, temperatura mínima promedio de $18,1^{\circ} \mathrm{C}$, máxima promedio de $31,2^{\circ} \mathrm{C}$, precipitación promedio anual de $1984 \mathrm{~mm}, c a .640 \mathrm{~m}$ de altitud (Vives 1973), una zona casi exclusivamente

\footnotetext{
${ }^{1}$ Morales, C.O. 1989. Fenología y producción de frutos y semillas del guanacaste, Enterolobium cyclocarpum (Jacq.) Griseb. (Leguminosae), en Turrúcares, Alajuela. Investigación por tutoría, guiada por A. Castaing Riba. Escuela de Biología, Universidad de Costa Rica.
} 
ganadera]. Como parte de ese estudio, entre marzo y abril de 1988 recolecté, de 13 árboles de guanacaste, 740 frutos maduros, secos, caídos, que se guardaron en bolsas plásticas cerradas, en una habitación con temperatura ambiente (promedio entre 20 y $25^{\circ} \mathrm{C}$ ). De cada árbol recogí un mínimo de 20 y un máximo de 100 frutos, dependiendo de la cosecha. Cuatro meses después de la recolecta, separé aleatoriamente y numeré 450 frutos de la muestra total. Medí el ancho máximo $(\mathrm{cm})$ como un parámetro del tamaño de la vaina. Dos meses después, escogí al azar el 50\% de los frutos numerados $(\mathrm{n}=225$, i.e. $30 \%$ de la muestra inicial), para determinar la masa de frutos y semillas y hacer observaciones sobre el estado fitosanitario de las semillas.

El estudio reveló un coeficiente de correlación significativamente alto $(r=0,95)$ entre la masa del fruto y la masa de las semillas viables. Éstas se distinguen fácilmente de las no viables (subdesarrolladas o dañadas) por tener una forma más regular, ser menos aplanadas, tener mayor brillo en la testa y mayor volumen de material de reserva.

La masa promedio de las semillas viables fue de $0,7 \pm 0,1 \mathrm{~g}(\mathrm{n}=2576)$, la de semillas dañadas por insectos $0,39 \pm 0,01 \mathrm{~g}(\mathrm{n}=24)$ y la de semillas subdesarrolladas $0,23 \pm 0,01 \mathrm{~g}(\mathrm{n}=27)$. Una prueba de chi cuadrado mostró que la proporción entre estos tres valores de masa es cercana a 3:2:1, respectivamente ( $\mathrm{p}>0,3,2$ grados de libertad).

El porcentaje de semillas dañadas por insectos o subdesarrolladas no superó el 5\% del total de la muestra. Janzen (1969, 1991) comunica valores mayores de masa de semillas (hasta 1,10 g) en la provincia de Guanacaste, Costa Rica (ámbito: 0,30 a 1,10 g, Janzen 1991), lo que parece sugerir que existen diferencias regionales en el desarrollo de las semillas de E. cyclocarpum. Yo deduzco que la masa óptima de semillas seleccionadas en Costa Rica, para propósitos de reproducción y propagación de esta especie, debe estar entre 0,7 y $1,0 \mathrm{~g}$.

Se hizo un análisis de variancia y regresión con los datos de frutos y semillas, que reveló una fuerte relación de causa-efecto entre masa de semillas viables y masa del fruto $(n=224$ frutos, 2576 semillas, $\mathrm{p}=1 \%$ y $5 \%$, grados de libertad 1 e infinitos). Además, este análisis indicó que la masa del fruto no depende del número de semillas viables. La fórmula obtenida es:

$$
\mathrm{M}=\mathbf{5 , 3 7}+\mathbf{2 , 0 0 m}
$$

Donde $\mathbf{M}$ es la masa del fruto (g) y m la masa de todas las semillas viables del fruto (g). Aquí se observa que la masa de las semillas viables explica una parte significativa de la variación en la masa del fruto. De este modo, se puede predecir que los frutos con mayor masa, más grandes y mejor formados, podrán contener semillas más voluminosas que, probablemente, serán las más viables $\mathrm{y}$, por consiguiente, tendrán mejores posibilidades de germinar rápidamente o de permanecer intactas en reposo durante cierto tiempo.

Además, se determinó que la anchura máxima del fruto no se correlaciona significativamente con la masa del fruto $(\mathrm{r}=0,64)$; tampoco con la masa de las semillas viables $(r=0,57)$ ni con el número de semillas por fruto $(r=0,44)$. Por ello, puedo argumentar que la anchura máxima no es un buen parámetro del tamaño del fruto, que a menudo se arquea a lo ancho cuando madura. Janzen (1982) utilizó el número de semillas viables (filled seeds) como indicador del tamaño del fruto; sin embargo, sería más conveniente considerar el número total de semillas, porque cada semilla, independientemente de su desarrollo o estado fitosanitario, ocupa un espacio dado dentro del fruto.

\section{Semillas de Enterolobium cyclocarpum en} reposo durante 13 años. Conservé las semillas secas, del estudio referido, en una bolsa plástica cerrada. En septiembre de 1994 las lancé en un terreno del distrito central de Desamparados, San José, Costa Rica (1150 m de altitud), junto a un muro de concreto, en un área pequeña de $c a .1,5 \mathrm{~m}$ x 0,3 m. El suelo es de relleno, en gran parte arcilloso pero con abundante arena y piedra menuda, expuesto a los rayos solares de la tarde (desde el oeste y el sudoeste) y a las lluvias del período húmedo del año. Las semillas quedaron allí parcial o completamente enterradas. Entre 1994 y 1998 no pude realizar observaciones; sin embargo, al caer los primeros aguaceros de la estación lluviosa de 1999 germinaron cerca de 10 semillas. En el 2000 germinó de nuevo un número similar de semillas. Entre febrero y abril de 2001 germinaron unas cinco semillas, 13 años después de haber sido recolectadas. Estas semillas germinaron antes del inicio de la estación lluviosa, debido a que llovió varias veces en San José durante la estación seca (diciembre a abril). Coloqué algunos arbolitos en bolsas y en macetas y los llevé al invernadero del Jardín Botánico Orozco, Montes de 
Oca, San José (1160 m de altitud). Desafortunadamente, todos fueron atacados por hongos que tornan blancos los tallos inmaduros y las hojas y rápidamente provocan pudrición. Al parecer, este invernadero era demasiado umbroso para plántulas que, como muchas del Pacífico Seco, necesitan espacios abiertos, con gran radiación solar, para crecer bien. El arbolito más robusto se plantó en junio de 1999 en un sitio abierto del campo del jardín referido, donde murió cerca de dos años más tarde. Otro arbolito, de las últimas semillas que germinaron en 2001, es el único que ha sobrevivido y crece exitosamente. Ahora sabemos que al menos algunas semillas de guanacaste pueden sobrevivir 13 años, probablemente mayor tiempo, en el suelo o en condiciones artificiales, hasta que, finalmente, la testa se rompe y ocurre germinación. Ésta será normal si en el microambiente no se hallan hongos, otros patógenos o herbívoros que pueden destruir un porcentaje alto de las semillas germinadas y las plántulas.

Un experimento fallido pero revelador. En julio de 2000 recibí 100 semillas viables del Dr. Luis Fournier Origgi, quien las había guardado durante 10 años, recogidas de árboles de guanacaste de Ciudad Colón, San José (ca. $800 \mathrm{~m}$ de altitud). En julio de 2001 (estación lluviosa) se diseñó un experimento sencillo para probar la capacidad de estas semillas para germinar.

1) Veinticinco semillas fueron limadas, con una lima de hierro aplicada vigorosamente en dos puntos opuestos, laterales, de cada semilla, hasta romper completamente la testa, pudiendo verse la parte interna, que es más clara.

2) Veinticinco semillas se sumergieron en agua a 45 ${ }^{\circ} \mathrm{C}$ durante cinco minutos.

3) Veinticinco semillas solamente se sumergieron en agua a temperatura ambiental (promedio: $24^{\circ} \mathrm{C}$ ) durante 24 horas. Luego se trasladaron al campo en el Jardín Botánico Orozco, donde fueron colocadas en suelo orgánico, parcialmente enterradas.

4) Veinticinco semillas no recibieron ningún tratamiento. Se colocaron en el campo del Jardín Botánico Orozco levemente cubiertas de tierra negra, orgánica.

Tomé la decisión de no utilizar ningún producto químico para proteger las semillas de patógenos, en caso de que germinaran. Mi idea era observar el proceso tan naturalmente como fuera posible. A posteriori, esta decisión puede considerarse errónea, por los resultados descritos a continuación.

Todas las semillas limadas o calentadas se colocaron en camas de germinación dentro del invernadero del Jardín Botánico Orozco, parcialmente enterradas en un suelo negro, orgánico, con irrigación manual. Todas sufrieron rápidamente el fenómeno de imbibición. Al romperse y prácticamente desintegrarse la testa de algunas de las semillas, los cotiledones se observaron normales, con un color verde pálido, amarillento, lo que parecía indicar que al menos varias de las semillas podían germinar sin problemas. Sin embargo, cuatro días después se observó un fuerte ataque fúngico en las semillas del invernadero (limadas o calentadas) y siete días después todas estaban perdidas. El ataque fúngico tornó todos los tejidos seminales blancos y realmente los desintegró en varios días.

Después de siete días y varios aguaceros, ca. 50\% de semillas sin ningún tratamiento, colocadas en el campo, estaban imbibidas y con la testa rota, mientras que la mayor parte de las semillas que habían sido sumergidas en agua a temperatura ambiental todavía no estaban imbibidas. En ambos casos, no hubo indicios de germinación. Sin embargo, a doce días del inicio se observó que cerca del 50\% de las semillas que habían sido sumergidas en agua a temperatura ambiental mostraban imbibición y un fuerte ataque fúngico, mientras que el resto no mostraba ningún cambio de color o forma (i.e. no sufrieron imbibición). Además, el 50\% de las semillas sin tratamiento ya estaban podridas, al ser atacadas por hongos, en tanto que el resto de las semillas no mostraba ningún cambio. Cinco meses después del inicio (diciembre de 2001) no fue notable ningún cambio de lo descrito sobre las semillas colocadas en el campo. A mediados de mayo de 2002 observé que, después de los primeros aguaceros de esa época lluviosa, varias semillas con y sin tratamiento habían sufrido imbibición, pero estaban podridas, prácticamente desintegradas por hongos. Un año y medio después del inicio del experimento (diciembre 2002), el resto de las semillas en el campo, que representan ca. $25 \%$ del número inicial de cada tratamiento, permanecían intactas en el suelo, sin imbibición, sin daño por hongos ni cambio de color, forma o volumen. 
Probable patrón de germinación de semillas de Enterolobium cyclocarpum. Lo que he observado hasta ahora me motiva a sugerir lo siguiente: Cuando llega la época lluviosa, no todas las semillas están preparadas para germinar, sino solamente cierta proporción de la cosecha. El resto de las semillas puede germinar en varios o numerosos períodos posteriores. Probablemente sólo cierto porcentaje de semillas sobrevive más allá de cinco años. Al mismo tiempo, en ciertas condiciones ambientales (como las de mi experimento fallido) se observa una alta tasa de mortalidad, porque muchas de las semillas que sufren imbibición pronto son atacadas por hongos que las destruyen rápidamente. Según las evidencias preliminares, cada semilla de E. cyclocarpum está programada para germinar en determinado momento después de madurar. En términos concretos, la ruptura de la testa ocurrirá de modo diferencial entre semillas, porque es probable que los procesos fisiológicos internos, que desencadenan respuestas a algunos factores ambientales, no ocurran al mismo tiempo en todas las semillas. Si las semillas de una cosecha determinada están programadas para germinar tanto de inmediato (con las primeras lluvias) como un año, dos años, hasta 10 o más años después de madurar, aquí se manifiesta una estrategia reproductiva ventajosa, especialmente si las condiciones ambientales se tornan adversas en algún lapso del ciclo de vida del árbol madre. ¿Cuáles son las condiciones microambientales óptimas para alcanzar el máximo reposo en semillas de guanacaste? Será necesario investigar mucho más la germinación y el reposo en esta especie.

A menudo se argumenta que en zonas tropicales las semillas están diseñadas para aprovechar condiciones más bien favorables; es decir, no pasan por un período de reposo, sino que tras madurar germinan tan pronto como caen (o poco tiempo después). No obstante, las semillas de Enterolobium cyclocarpum pueden estar en reposo y ser viables durante largo tiempo. El reposo de semillas (llamado también letargo, latencia o dormancia) se debe a fenómenos mecánicos o fisiológicos que impiden el crecimiento del embrión, incluso en condiciones apropiadas, y tiene implicaciones evolutivas y adaptativas ( $c f$. Flores 1989, Janzen \& Wilson 1974). Yo considero que en el guanacaste la resistencia mecánica y la impermeabilidad de la testa son factores determinantes del reposo; sin embargo, la influencia de factores fisiológicos, genéticos y ambientales no puede descartarse ( $c f$. Flores 1989). Strasburger (1994: 451) señala que semillas de vida larga se encuentran sobre todo en leguminosas, malváceas, en el loto de China (Nelumbo nucifera Gaertn., Nymphaeaceae), cuyas semillas se ha comprobado que pueden sobrevivir hasta mil años, y en otras especies. Las semillas de muchas malas hierbas, en ausencia de oxígeno, pueden permanecer en reposo durante siglos. Por otro lado, el trigo mantiene su capacidad germinativa no más de 10 años; por tanto, es falsa la noticia sobre la germinación de trigo hallado en antiguas tumbas egipcias. Las semillas de plantas tropicales generalmente no están adaptadas para sobrepasar períodos tan desfavorables como el invierno de las zonas templadas y a menudo sobreviven menos de un año.

\section{Regeneración natural y sobrevivencia del} guanacaste. En Turrúcares, después del inicio de las lluvias, bajo un árbol de guanacaste conté 80 plántulas y semillas en germinación de esta especie por $\mathrm{m}^{2}$, creciendo principalmente en estiércol bovino. Esto último parece confirmar observaciones anteriores ( $c f$. Holdridge et $a l$. 1997, Janzen 1982) en el sentido de que las semillas germinan más fácilmente después de haber pasado (dentro del fruto) por el sistema digestivo de mamíferos grandes. Bajo otro árbol de guanacaste medí arbolitos de hasta 28 $\mathrm{cm}$ de altura, creciendo también en gran densidad. Se observó que prácticamente todas las semillas viables, que eran visibles, ya habían germinado o empezaban a sufrir imbibición. Varios meses después no quedó ni una sola plántula de guanacaste viva, debido a una combinación de factores: la sombra de la vegetación que reverdece al iniciarse la estación lluviosa, el regreso del ganado a estas áreas de pastoreo en la estación lluviosa, el ataque de patógenos, la competencia de gramíneas y otras hierbas, la gran densidad de los mismos arbolitos y la sequedad del suelo entre diciembre y mayo. En la época seca muere la mayoría de arbolitos por falta de agua o por herbivoría, sobre todo en bosques secos, donde la mayor parte de la vegetación pierde el follaje, o en zonas ganaderas. Así, la reproducción natural de $E$. cyclocarpum es muy baja en Costa Rica, hasta el punto de que Janzen (1991) supone que en un siglo esta especie se extinguirá en ambientes naturales de Costa Rica.

Al mismo tiempo, es evidente que esta especie 
tiene un gran potencial reproductivo, que puede aprovecharse racionalmente. En condiciones ambientales favorables, el guanacaste crece relativamente rápido, puede regenerarse en bosques secundarios protegidos, ofrece sombra a otras especies vegetales y al ganado, contribuye en la fijación de nitrógeno atmosférico y es un elemento significativo y emblemático del paisaje, sobre todo en las regiones Pacífico Norte (provincia de Guanacaste) y Pacífico Central de Costa Rica. Además, en sistemas silviculturales puede proporcionar madera de gran utilidad para carpintería y ebanistería ( $c f$. Bertoni \& Juárez 1980, Bertsch 1985, Camacho 1981, Fournier 1977, González 1980, Janzen 1991, Record \& Hess 1949, entre otros). Una mayor comprensión de la biología reproductiva y de los factores que afectan el crecimiento de las especies arbóreas tropicales será crucial para protegerlas y aprovecharlas de modo verdaderamente sostenible.

AGRADECIMIENTOS. Mi gratitud hacia el profesor Álvaro Castaing Riba es perenne, pues él fue para mí un guía, un maestro y un amigo al mismo tiempo. En mi memoria permanecen, como recuerdo imborrable, las lecciones y la sabiduría del finado Luis Fournier Origgi (ver LANKESTERIANA 5), el eminente ecólogo que se interesó en mi investigación.

\section{LITERATURA CITADA}

Bertoni V., R. \& Juárez G., V.M. 1980. Comportamiento de nueve especies forestales tropicales plantadas en 1971 en el Campo Experimental Forestal Tropical "El Tormento". Ciencia Forestal 5(25): 3-40.

Bertsch, Sonia. 1985. Germinación y crecimiento del guanacaste (Enterolobium cyclocarpum (Jacq.) Griseb.) en vivero y en el campo. Tesis de Licenciatura. Escuela de Biología, Universidad de Costa Rica. San José. 68 p.

Camacho M., P. 1981. Ensayos de adaptabilidad y rendimiento de especies forestales en Costa Rica. Cartago, ITCR / MAG. p. 29-31.
Duarte, A.P. 1978. Contribuição ao conhecimento da germinação de algumas essências florestais. Rodriguésia 30(45): 439-446.

Flores V., Eugenia. 1989. La Planta, estructura y función. Cartago, Edit. Tecnológica de Costa Rica. 501 p.

Fournier O., L.A. 1977. La sucesión ecológica como un método eficaz para la recuperación del bosque en Costa Rica. Agron. Costar. 1(1): 23-29.

González, R. 1980. Plantaciones forestales a nivel experimental en Costa Rica. Agron. Costar. 4(1): 99109.

Holdridge, L.R., Poveda, L.J. \& Jiménez, Q. 1997. Árboles de Costa Rica. Vol. 1, 2 ${ }^{\text {a }}$. ed., actualizada por Q. Jiménez. San José, Centro Científico Tropical. 522 p.

Janzen, D.H. 1969. Seed-eaters versus seed size, number, toxicity and dispersal. Evolution 23(1): 1-27.

Janzen, D.H. 1982. Variation in average seed size and fruit seediness in a fruit crop of a guanacaste tree (Leguminosae: Enterolobium cyclocarpum). Amer. J. Bot. 69(7): 1169-1178.

Janzen, D.H. 1991. Enterolobium cyclocarpum (Leguminosae) (Guanacaste, Ear Tree). In: Janzen, D.H. (ed.). Historia Natural de Costa Rica. Trad. M. Chavarría A. San José, Edit. Universidad de Costa Rica. p. 244-246.

Janzen, D.H. \& Wilson, D.E. 1974. The cost of being dormant in the tropics. Biotropica 6: 260-262.

Record, S.J. \& Hess, R.W. 1949. Timbers of the New World. 4ta. impr. Yale University Press. p. 266.

Strasburger, E. 1994. Tratado de Botánica. $8^{\text {a }}$. edic. castellana de la $33^{\mathrm{a}}$. edic. alemana de Lehrbuch der Botanik, E. Strasburger, F. Noll, H. Schenck \& A.F.W. Schimper, actualizada por P. Sitte, H. Ziegler, F. Ehrendorfer \& A. Bresinsky. Barcelona, Ediciones Omega. 1068 p.

Vásquez-Yanes, C. \& Pérez-García, Blanca. 1977. Notas sobre la morfología, la anatomía de la testa y la fisiología de las semillas de Enterolobium cyclocarpum. Turrialba 27(4): 427-430.

Vives F., L.A. 1973. Tabulación para uso agrícola de los datos climáticos de Costa Rica. San José, Facultad de Agronomía, Universidad de Costa Rica. 222 p. 